\title{
Low Dose of Lenvatinib Treatment for Patients of Radioiodine-Refractory Differentiated Thyroid Carcinoma - A Real-World Experience
}

\author{
He-jiun Jiang' \\ Yen-Hsiang Chang ${ }^{2}$ \\ Yen-Hao Chen ${ }^{3}$ \\ Che-Wei Wu (D) ${ }^{4}$ \\ Pei-Wen Wang ${ }^{5}$ \\ Pi-Jung Hsiao (D) ${ }^{6}$
}

'Division of Endocrinology and Metabolism, Department of Internal Medicine, E-DA DaChang Hospital, Kaohsiung, Taiwan; ${ }^{2}$ Department of Nuclear Medicine, Kaohsiung Chang Gung Memorial Hospital, Kaohsiung, Taiwan; ${ }^{3}$ Department of HematologyOncology, Kaohsiung Chang-Gung Memorial Hospital, Chang Gung University, Kaohsiung, Taiwan; ${ }^{4}$ Department of Otolaryngology-Head and Neck Surgery, Kaohsiung Medical University, Kaohsiung, Taiwan; ${ }^{5}$ Division of Endocrinology and Metabolism, Department of Internal Medicine; Department of Nuclear Medicine; Kaohsiung Chang-Gung Memorial Hospital, Chang Gung University, Endocrinology and Metabolism, Hospital; Thyroid Center, E-DA Cancer Hospital; College of Medicine, I-Shou University, Kaohsiung, Taiwan Kaohsiung, Taiwan; ${ }^{6}$ Division of Department of Internal Medicine, E-DA

Background: Lenvatinib treatment of $24 \mathrm{mg} /$ day for radioiodine-refractory differentiated thyroid carcinoma (RRDTC) patients was almost intolerable, with high rates of dose reduction, interruption and discontinuation. Balancing treatment safety with disease risks remains challenging, and the appropriate dosage remains unclear in Asia.

Patients and Methods: A total of 65 RRDTC patients treated with lenvatinib were retrospectively collected from Oct. 2015 to Jun. 2020 from two medical centers of South Taiwan. The drug tolerability, treatment efficacy and clinical outcomes were analyzed.

Results: Different doses of lenvatinib were initiated but ultimately maintained with a median dose of $10 \mathrm{mg} /$ day within the first 3 months. The disease control rate reached $89.2 \%$, including $24.6 \%$ partial response and $64.6 \%$ stable disease. Disease progression occurred in $10.8 \%$ of patients and increased to $40.0 \%$ at the end. Eventually, the treatment dose achieved a median progression-free survival (PFS) of 26.1 months (95\% CI: 17.1-NA) with overall survival (OS) not reached yet (24.1 NA). Overall, the 48-month PFS rate was $35.6 \%$ (95\% CI: 18.5-68.4) and 48-month OS was 54.3\% (95\% CI: 41.2-71.7). The dose was tolerable with a dose reduction rate of $44.6 \%$, dose interruption rate of $40.0 \%$ and fewer high-graded adverse events. The drug discontinuation rate was only $3.1 \%$. However, RRDTC patients with bone metastasis or maximal dose exposure to RAI ( $\geq 600 \mathrm{mCi}$ ) may have less efficacy to the low maintenance dose treatment.

Conclusion: Assessing treatment intensity, safety and efficacy, low-dose lenvatinib treatment was well tolerated by RRDTC patients and displayed acceptable drug efficacy and outcomes.

Keywords: lenvatinib, radioiodine-refractory differentiated thyroid carcinoma, RRDTC, disease control rate, DCR, progression-free survival, PFS, overall survival, OS

\section{Plain Language Summary}

1. The low maintenance dose of lenvatinib (10 mg/day) was well tolerated for patients of radioiodine-refractory differentiated thyroid carcinoma with relatively less dose reduction, interruption, drug discontinuation and minor grades of adverse events.

Correspondence: Pi-Jung Hsiao Division of Endocrinology and Metabolism, Department of Internal Medicine, E-DA Hospital; Thyroid Center, E-DA Cancer Hospital; College of Medicine, I-Shou University, No. I, Yida Road, Yanchao District, Kaohsiung, 82445, Taiwan

Tel +886-7-6I500II ext 2325

Fax +886-7-558142I

Email pjhsiaol0I@gmail.com
2. The low maintenance dose lenvatinib treatment could achieve disease control rate of $89.2 \%$ and median progression-free survival of 26.1 months.

3. Patients with radioiodine-refractory differentiated thyroid carcinoma with bone metastasis or maximal dose exposure to RAI ( $\geq 600 \mathrm{mCi}$ ) may have less efficacy to the low maintenance dose treatment.

4. The initial drug response to lenvatinib at first 3 months could predict the outcome well. 


\section{Introduction}

The incidence of thyroid cancer continues to rise worldwide. Generally, most differentiated thyroid carcinoma (DTC) is near-curable after surgery combined with radioiodine (RAI) therapy. However, disease persistence and recurrence are reported up to $30 \%{ }^{1,2}$ And $10 \sim 15 \%$ of these patients may progress to advanced stage with local invasion and/or distant metastasis. Concurrently, 25 50\% of these patients are refractory to RAI therapy; then, the management becomes very challenging. Overall, patients with RAI-refractory DTC (RRDTC) are estimated 4-5 cases/million/year. Afterward, the 10-year survival drops to $10 \%$ with a life expectancy by $3-5$ years. ${ }^{3-6}$

Lenvatinib, an approved multi-kinase inhibitor (TKI), significantly prolongs median progressive-free survival (PFS, 18.3 vs 3.6 months of placebo) for RRDTC patients. ${ }^{7-10}$ The Phase II study of lenvatinib treatment determined a maximal tolerated dose of $25 \mathrm{mg} /$ day and initiated a dose of $24 \mathrm{mg} /$ day. ${ }^{11}$ However, the incidence of adverse events (AEs) of any grade and higher grade were $97.3 \%$ and $75.9 \%$, respectively, which definitely restricted maintenance of the standard dose. The dose reduction or interruption was reported to be 59.0 $\sim 73.6 \%$ and $31.0 \sim 86.8 \%$ in clinical reality. ${ }^{8,12}$ The Japanese studies ever reported significant higher liver toxicities and higher $(\sim 90 \%)$ discontinuous rate for a standard dosage of $24 \mathrm{mg} /$ day lenvatinib. ${ }^{7,9,13,14}$ Therefore, balancing treatment intensity, safety, and the risk posed by the disease remains challenging. Especially, it is still unclear how to manage the tolerably effective dose for Asian population.

Thus, a retrospective observational study from two medical centers in South Taiwan was conducted, where all RRDTC patients were treated with a low dose of lenvatinib. We assessed this real-world experience for drug efficacy and outcome by disease control rate (DCR), median PFS, and overall survival (OS). Our results demonstrated that the low dose ( $\sim 10 \mathrm{mg} /$ day) of lenvatinib treatment was well tolerated for RRDTC patients to achieve acceptable outcome with relatively less dose reduction, interruption, drug discontinuation and minor grades of AE.

\section{Patients and Methods}

This study was conducted after approval by the Institutional Review Boards of both medical centers (KMUHIRB-E (I)20190014 and CGMHIRB No. 201801270B0). Since this study was a retrospective observational study without any intervention, the clinical dataset was permitted for review without informed consent from patients of advanced
RRDTC patients treated with lenvatinib at Kaohsiung Medical University and Kaohsiung Chang-Gung Memorial Hospital from Oct.2015 to Jun. 2020. All of the medical record reviews were carried out confidentially to comply with the Declaration of Helsinki. The study included 65 DTC patients who all met the RRDTC criteria with cumulated doses of radioiodine therapy $\geq 600 \mathrm{mCi}$, presence of any RAI refractory new lesion or RAI-avid lesion with progressive enlargement.

The medical record was independently reviewed by two authors, including the patient's Eastern Cooperative Oncology Group (ECOG) performance status, pathology, AEs, lesion or disease progression by imaging evidences, and drug efficacy. For consistent reporting between hospitals, all the image studies for tumor assessment from radioiodine whole body scan, computerized tomography, bone scan, magnetic resonance image or positron emission tomography were reviewed again and standardized according to the Response Evaluation Criteria in Solid Tumors (RECIST) version 1.1 by one professional nuclear medicine radiologist. The drug response was assessed by image evidences at the first 3 months of lenvatinib treatment and then kept with an interval of $3 \sim 6$ months. The AEs were recorded following the National Cancer Institute Common Terminology Criteria for Adverse Events (CTCAE) version 5.0. Treatment timeline of the study population was illustrated using R4.0.2 (R Core Team, 2020). All patients were initially tracked from the starting date of lenvatinib treatment, and the time point of PD, death or censor were marked according to the date of PD documented, death date or end date of follow-up, respectively.

\section{Statistical Analysis}

The statistical analyses were performed with Stata version 14.0. (StataCorp. 2015. Stata Statistical Software: Release 14. College Station, TX: StataCorp LP). The continuous variables were indicated as frequency (percentage), mean (standard deviation) or median (interquartile range, IOR). The differences of categorical variables among groups were estimated by Fisher's exact test. For continuous variables, the differences among groups were estimated using KruskalWallis test, and the Mann-Whitney $U$-test was used in pairwise comparison for each group. Median PFS and OS of each group were reported with 95\% confidence intervals (CIs), and the survival curve of all patients and group stratification were illustrated by Kaplan-Meier estimator. The survival differences between groups were estimated by the Log rank test. $P$ values less than 0.05 were considered statistically significant. 


\section{Results}

Patients enrolled in this study were followed from 0.4 to 48 months. The median follow-up period was 17.1 months, and the treatment timeline of all patients is shown in Supplementary Figure 1. Most of the recruited patient (85.4\%) presented with ECOG performance score $0-1$ before initiation of lenvatinib treatment. Lung was primarily documented to be the most common metastatic lesions, followed by neck and bone. Advanced locoregional invasion to adjacent critical organs, including carotid artery, jugular vein, trachea or esophagus, occurred in $30.8 \%$ of the patients. Some patients $(21.5 \%)$ were previously exposed to other TKI therapy before lenvatinib (Table 1).

Table I Clinicopathological Characteristics Before Initiation of Lenvatinib

\begin{tabular}{|l|c|}
\hline Baseline Characteristics & $\mathbf{n ~ ( \% )}$ \\
\hline Cases no. & 65 \\
\hline Age (years), mean \pm SD & $65 \pm 12$ \\
\hline Sex, female & $30(46.2 \%)$ \\
\hline BW (kg), mean \pm SD & $63.4 \pm 13.3$ \\
\hline Histology & \\
Papillary carcinoma & $55(84.6 \%)$ \\
Follicular carcinoma & $9(13.8 \%)$ \\
Hürthle cell carcinoma & $1(1.5 \%)$ \\
\hline ECOG before lenvatinib & $17(26.2 \%)$ \\
0 & $40(61.5 \%)$ \\
I & $5(7.7 \%)$ \\
2 & $2(3.1 \%)$ \\
3 & $1(1.5 \%)$ \\
4 & \\
\hline Metastatic lesions involved & $54(83.1 \%)$ \\
Lung & $25(38.5 \%)$ \\
Bone & $38(58.5 \%)$ \\
Neck & $20(30.8 \%)$ \\
\hline Cuther sites a & $400(300-540)$ \\
\hline Subgroups of RRDTC ${ }^{b}$ & $28(20.0 \%)$ \\
PreomCi & $14(21.5 \%)$ \\
Disease progression in RAl avid lesion & \\
\hline Previous another TKI use before lenvatinib & \\
\hline & \\
\hline
\end{tabular}

Notes: ${ }^{a}$ Other sites involved included metastasis to brain, liver, and skin, etc. ${ }^{\mathrm{b}} \mathrm{RRDTC}$ indicated radioiodine refractory differentiated thyroid carcinoma.

Abbreviations: SD, standard deviation; IQR, interquartile range.
The lenvatinib treatment was differently initiated with doses ranged 4 to $24 \mathrm{mg} / \mathrm{d}$, which was determined by individual physician (Supplementary Figure 1). The median dose was $10 \mathrm{mg}$ /day with an interquartile range (IOR) of 10 14 mg/day within first 2 4-week. The dose titration was almost achieved a tolerable maintenance dose within the first month. The median maintenance dose was eventually around 10 (IOR, 8 14) mg/day until the 3rd month of treatment, with AE-related dose reduction and interruption in $44.6 \%$ and $40.0 \%$, respectively (Table 2 ). However, lenvatinib was discontinued in 2 patients $(3.1 \%)$ because of rapid disease progression and they all died soon after.

At the initial 3 months, the DCR reached $89.2 \%$, including $24.6 \%$ of partial response (PR) and $64.6 \%$ of stable disease (SD). Progressive disease (PD) occurred in $10.8 \%$ and reached $40.0 \%$ of patients at the end of follow (Table 2). Ultimately, the median PFS was 26.1 months (17.1 not available (NA)) and the median OS has not reached (Figure 1). Overall, the 48-month PFS was $35.6 \%(95 \%$ CI: $18.5-68.4)$ and 48-month OS was $54.3 \%$ (95\% CI: 41.2-71.7). Within period, 21 (32.3\%)

Table 2 Overall Efficacy and Tolerability of Low Maintenance Dose of Lenvatinib

\begin{tabular}{|l|c|}
\hline Overall Efficacy and Tolerability & n (\%) \\
\hline Drug dosage & $10(10-14)$ \\
Initial median dose (mg/d), median (IQR) & $10(8-14)$ \\
Maintenance dose (mg/d), median (IQR) & $29(44.6 \%)$ \\
Dose reduction & $26(40.0 \%)$ \\
Dose Interruption & \\
\hline Initial drug response to lenvatinib & - \\
Complete response (CR) & $16(24.6 \%)$ \\
Partial response (PR) & $72(64.6 \%)$ \\
Stable disease (SD) & $7(10.8 \%)$ \\
Progressive disease (PD) & $58(89.2 \%)$ \\
Disease control rate (PR + SD) & $27(41.5 \%)$ \\
\hline Progression disease at the end of follow-up & $26.1(17.1-\mathrm{NA})$ \\
\hline PFS (months), median (95\% Cl) & NA (24.I-NA) \\
\hline OS (months), median (95\% Cl) & \\
\hline Overall deaths & $14(21.5 \%)$ \\
Disease related death & $3(4.6 \%)$ \\
Treatment related death ${ }^{\text {a }}$ & $4(6.2 \%)$ \\
Others related death ${ }^{\text {b }}$ & \\
\hline
\end{tabular}

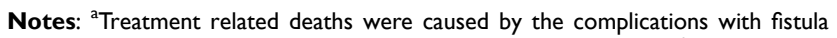
formation to the trachea, esophagus, carotid artery or jugular vein. ${ }^{\text {b Other related }}$ deaths were caused by cachexia and sepsis.

Abbreviations: IQR, interquartile range; PFS, progression free survival; OS, overall survival; $\mathrm{Cl}$, confidence interval; NA, not available or survival not reached yet. 


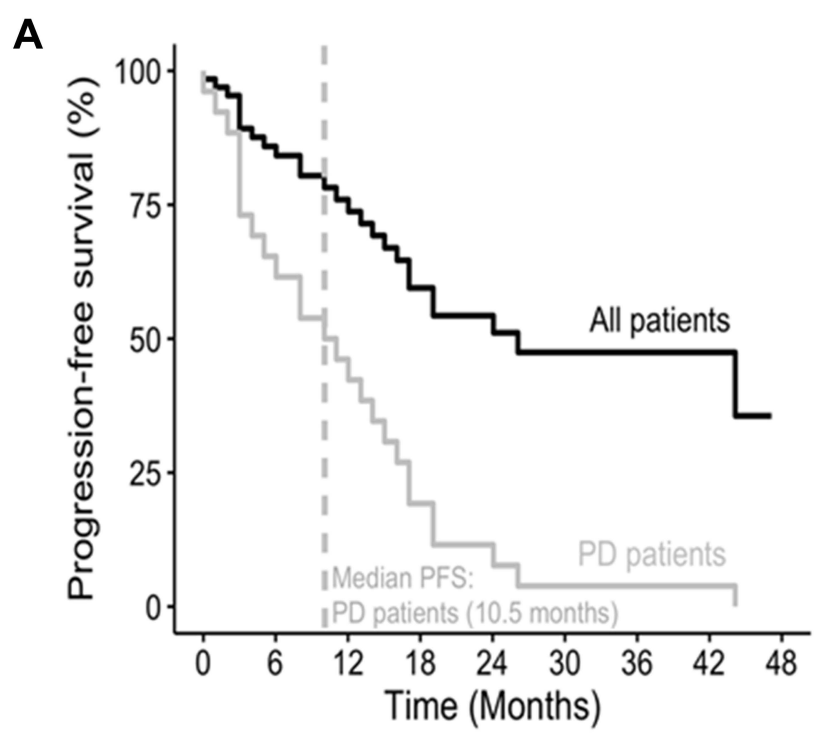

Number at risk
B

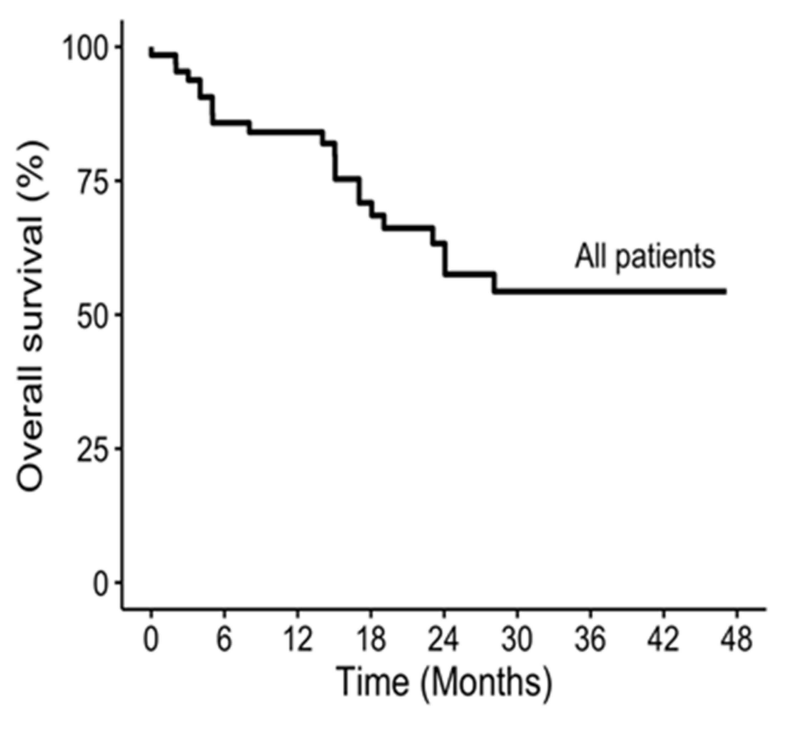

Number at risk

\section{All patients $65 \begin{array}{llllllll}60 & 34 & 23 & 17 & 12 & 12 & 6 & 0\end{array}$

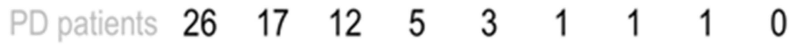 \\ All patients $\quad \begin{array}{lllllllll}65 & 53 & 40 & 30 & 22 & 16 & 16 & 9 & 0\end{array}$}

Figure I Kaplan-Meier curve of median progression-free survival (PFS) and overall survival (OS) in patients with advanced radioiodine-refractory differentiated thyroid carcinoma treated by low maintenance dose lenvatinib $(n=65)$. (A) The 48-month of PFS rate was $35.6 \%(95 \%$ Cl: 18.5-68.4) and median PFS was I0.5 months in group of progressive disease. (B) The 48-month of OS rate was $54.3 \%$ (95\% Cl: $41.2-71.7)$ in all patients.

patients died and most of them were caused by terminal RRDTC (Table 2).

The reported AEs mainly occurred in the first 3 months, which were primarily presented in low grade and rare with high grade. The AE associated dose reduction or interruption of lenvatinib was always resumed within days to maintain at tolerable levels until the end of follow-up. However, hypertension, asthenia and proteinuria were seen in over half of the patients (Supplementary Table 1).

In our study, the treatment response was widely distributed with a median PFS of 10.5 months in PD group. To further analyze the difference in treatment efficacy and outcome, we divided the PD patients into group 1: PFS $\leq 10.5$ months ( $\mathrm{n}=13)$, and group 2: PFS $>10.5$ months $(\mathrm{n}=13)$ as well as group 3: non-PD patients ( $n=39$ ). The ECOG status among 3 groups had no significant change before treatment. It was almost unchanged by low-dose lenvatinib treatment among the three groups (Supplementary Table 2). Patients ever exposed to maximal dose of RAI therapy ( $\geq 600 \mathrm{mCi}$ ) and those with lesion involved metastasis to bone and other sites (brain, liver and skin, etc.) occurred more in the PD than non-PD groups (Table 3). The initial dose and dose reduction rate were significantly higher in group 2 than the other groups. But the maintenance dose and dose interruption rate had no difference among the three groups (Table 4). However, the initial drug response displayed a significant discrimination at the first 3 months (Figure 2A). The group 1 patients displayed a significantly higher (53.8\%) PD and less PR (7.7\%) rates than the other groups. The initial drug response of SD was relatively less $(38.5 \%)$ in group 1 patients than the other groups $(84.6 \%$ of group 2 and $66.7 \%$ of group 3). Initial drug response to the low-dose lenvatinib treatment may predict future drug efficacy and outcome. Consequently, the group 1 patients had significantly worse PFS (3.0 months) and median OS (8.0 months) with greater mortalities $(69.2 \%)$ than the other groups (Table 4). The 48-month PFS among the 3 groups was 0,0 and $100 \%$, respectively. And the 48-month OS among 3 groups was 0, 41.7\% (95\% CI: $21.3-81.4$ ) and $81.7 \%$ (95\% CI: 67.5-98.9), respectively (Figure $2 \mathrm{~B}$ and C). Frequency of AEs was similar among groups in whole treatment course, but proteinuria (greater than grade 2) appeared more in group 2 than group 1 patients. Subanalysis of the proteinuria, including previously existing proteinuria, co-morbidities, incidental proteinuria or worsening of proteinuria after lenvatinib treatment, did not reveal any difference among the three groups. 
Table 3 Comparison of the Clinicopathological Characteristics Among Patients with PFS $\leq 10.5$ Months, PFS > 10.5 Months and NonPD Patients

\begin{tabular}{|c|c|c|c|c|}
\hline \multirow[t]{2}{*}{ Characteristics } & \multicolumn{2}{|c|}{ PD Group } & \multirow[t]{2}{*}{ Non-PD } & \multirow[t]{2}{*}{$\mathbf{P}$} \\
\hline & PFS $\leq 10.5 \mathrm{~m}$ & PFS $>10.5 \mathrm{~m}$ & & \\
\hline Case no. (row \%) & $13(20.0 \%)$ & $13(20.0 \%)$ & 39 (60.0\%) & \\
\hline Age (years), mean $\pm S D$ & $67 \pm 12$ & $67 \pm 11$ & $64 \pm 12$ & 0.523 \\
\hline Sex, female & $7(53.8 \%)$ & $6(46.2 \%)$ & $17(43.6 \%)$ & 0.814 \\
\hline $\mathrm{BW}(\mathrm{kg})$, mean $\pm \mathrm{SD}$ & $59.5 \pm 15.9$ & $63.5 \pm 12.4$ & $64.6 \pm 12.7$ & 0.524 \\
\hline $\begin{array}{l}\text { Histology } \\
\text { Papillary carcinoma } \\
\text { Follicular carcinoma } \\
\text { Hürthle cell carcinoma }\end{array}$ & $\begin{array}{c}\text { II (84.6\%) } \\
2(15.4 \%) \\
-\end{array}$ & $\begin{array}{c}10(76.9 \%) \\
2(15.4 \%) \\
1(7.7 \%)\end{array}$ & $\begin{array}{c}34(87.2 \%) \\
5(12.8 \%) \\
-\end{array}$ & 0.568 \\
\hline $\begin{array}{l}\text { ECOG change from baseline } \\
\text { Improved } \\
\text { Unchanged } \\
\text { Worsened }\end{array}$ & $\begin{array}{c}\text { I (7.7\%) } \\
\text { II (84.6\%) } \\
\text { I (7.7\%) }\end{array}$ & $\begin{array}{l}\text { I (7.7\%) } \\
\text { I I (84.6\%) } \\
\text { I (7.7\%) }\end{array}$ & $\begin{array}{c}2(5.1 \%) \\
30(76.9 \%) \\
7(17.9 \%)\end{array}$ & 0.830 \\
\hline $\begin{array}{l}\text { Lesion involvement } \\
\text { Lung } \\
\text { Bone } \\
\text { Neck } \\
\text { Other sites }\end{array}$ & $\begin{array}{l}\text { II (84.6\%) } \\
9(69.2 \%) \\
9(69.2 \%) \\
6(46.2 \%)\end{array}$ & $\begin{array}{l}12(92.3 \%) \\
6(46.2 \%) \\
8(61.5 \%) \\
8(61.5 \%)\end{array}$ & $\begin{array}{l}31(79.5 \%) \\
10(25.6 \%)^{a} \\
21(53.8 \%) \\
6(15.4 \%)^{b}\end{array}$ & $\begin{array}{l}0.733 \\
0.016 \\
0.689 \\
0.003\end{array}$ \\
\hline Cumulative radioiodine dose, median (IQR) & $375(237.5-487.5)$ & $570(4|2.5-6| 2.5)$ & $400(300-500)$ & 0.135 \\
\hline $\begin{array}{l}\text { Subgroup of RRDTC } \\
\geq 600 \mathrm{mCi} \\
\text { Presence of any RAI refractory lesion } \\
\text { Disease progression in RAl avid lesion }\end{array}$ & $\begin{array}{l}3(23.1 \%) \\
8(61.5 \%) \\
9(69.2 \%)\end{array}$ & $\begin{array}{l}6(46.2 \%) \\
9(69.2 \%) \\
5(38.5 \%)\end{array}$ & $\begin{array}{l}4(10.3 \%) \\
28(71.8 \%) \\
14(35.9 \%)\end{array}$ & $\begin{array}{l}0.016 \\
0.812 \\
0.129\end{array}$ \\
\hline Structural progression before lenvatinib & $13(100.0 \%)$ & $12(92.3 \%)$ & $28(71.8 \%)^{a}$ & 0.046 \\
\hline Previous another TKI use before lenvatinib & $4(30.8 \%)$ & $4(30.8 \%)$ & $6(15.4 \%)$ & 0.286 \\
\hline
\end{tabular}

Notes: $P$-value were estimated using Fisher's exact test and kruskal-Wallis test appropriately. ${ }^{a}$ Non-PD group obtained statistically significant lower proportion compared to group of PFS $\leq 10.5$ months. ${ }^{b}$ Non-PD group obtained statistically significant lower proportion compared to group of PFS $>10.5$ months.

Abbreviations: SD, standard deviation; IQR, interquartile range; RAI, radioiodine; RRDTC, radioiodine refractory differentiated thyroid carcinoma.

\section{Discussion}

TKI is used as a salvage therapy for advanced RRDTC patients. It is nearly impossible to achieve complete responses or tumor eradication. In fact, the median OS could not be significantly prolonged by TKI therapy in both the DECISION and SELECT trials. ${ }^{8,15}$ Therefore, it seems more practical to target prolongation of the median PFS, sustain a durable drug response and maintain an acceptable QoL by TKI therapy. Accordingly, optimization of TKI treatment should consider how to balance the efficacy, tolerability, risk posed by therapy and the impact on patient's QoL. ${ }^{16,17}$ Several factors have been reported to correlate with the response and outcome of lenvatinib treatment for RRDTC patients, such as initial dose, patient's age, patient's body weight, AEs or dose interruption. The SELECT Phase III trial initiating $24 \mathrm{mg} /$ day of lenvatinib for advanced RRDTC patients significantly improved PFS to achieve a $64.8 \%$ response rate and 87.7\% DCR but the discontinuation rate of lenvatinib was $14.2 \%{ }^{8}$ A retrospective review in France, mainly initiated lenvatinib $24 \mathrm{mg} /$ day, demonstrated a median PFS of 10 months and $82 \%$ DCR (PR, 31\% and SD, $51 \%$ ) with $14.7 \%$ of drug discontinuation. ${ }^{12}$ A retrospective report of 56 DTC patients with distant metastases showed a significantly extended SD period with the sorafenib or lenvatinib to reach $28.5 \%$ of response rate and the $75 \%$ of DCR. However, the median time of treatment failure and failure rate was 3.8 months and 
Table 4 Comparison of the Drug Efficacy, Tolerability, Adverse Events and Outcome Among Patients with PFS $\leq 10.5$ Months, PFS $>10.5$ Months and Non-PD Patients

\begin{tabular}{|c|c|c|c|c|}
\hline \multirow[t]{2}{*}{ Characteristics } & \multicolumn{2}{|c|}{ PD Group } & \multirow[t]{2}{*}{ Non-PD Patients } & \multirow[t]{2}{*}{$P$} \\
\hline & PFS $\leq 10.5 \mathrm{~m}$ & PFS $>10.5 \mathrm{~m}$ & & \\
\hline Case no. (row \%) & $13(20.0 \%)$ & $13(20.0 \%)$ & $39(60.0 \%)$ & \\
\hline \multicolumn{5}{|l|}{ Drug dosage } \\
\hline Initial median dose $(\mathrm{mg} / \mathrm{d})$, median (IQR) & $10(10-15)$ & $14(10-20)^{a}$ & $10(10-14)$ & 0.046 \\
\hline Maintenance dose (mg/d), median (IQR) & $10(10-15)$ & $10(5-14)$ & $10(9-14)$ & 0.246 \\
\hline Dose reduction & $4(30.8 \%)$ & $10(76.9 \%)^{a}$ & $15(38.5 \%)$ & 0.029 \\
\hline Dose interruption & $7(53.8 \%)$ & $7(53.8 \%)$ & $12(30.8 \%)$ & 0.177 \\
\hline Progression disease at the end of follow-up & $13(100.0 \%)$ & $13(100.0 \%)$ & - & $<0.001$ \\
\hline PFS (months), median $(95 \% \mathrm{Cl})$ & $3.0(3.0-\mathrm{NA})$ & I7.I (I4.0-NA) & NA (NA-NA) & $<0.001$ \\
\hline OS (months), median $(95 \% \mathrm{Cl})$ & $8.0(1.0-\mathrm{NA})$ & $24 . I$ (I8.I-NA) & NA (NA-NA) & $<0.001$ \\
\hline \multicolumn{5}{|l|}{ Overall deaths } \\
\hline Disease related death & $8(61.5 \%)$ & $6(46.2 \%)$ & - & $<0.001$ \\
\hline Treatment related death ${ }^{a}$ & I (7.7\%) & - & $2(5.1 \%)$ & $<0.001$ \\
\hline Others related death ${ }^{b}$ & - & I (7.7\%) & $3(7.7 \%)$ & 1.000 \\
\hline \multicolumn{5}{|l|}{ Severe adverse events (grade $\geq 2)^{c}$} \\
\hline Hypertension & $2(15.4 \%)$ & $4(30.8 \%)$ & $5(12.8 \%)$ & 0.373 \\
\hline Proteinuria & I (7.7\%) & $7(53.8 \%)^{d}$ & 7 (17.9\%) & 0.014 \\
\hline Gastrointestinal tract & - & I (7.7\%) & $2(5.1 \%)$ & 1.000 \\
\hline Fatigue & $3(23.1 \%)$ & I (7.7\%) & $2(5.1 \%)$ & 0.177 \\
\hline Weight loss & - & I (7.7\%) & $3(7.7 \%)$ & 0.815 \\
\hline Hand-foot syndrome & $4(30.8 \%)$ & $2(15.4 \%)$ & $3(7.7 \%)$ & 0.099 \\
\hline Anorexia & - & I (7.7\%) & $3(7.7 \%)$ & 0.815 \\
\hline
\end{tabular}

Notes: ${ }^{a}$ Treatment related deaths were caused by the complications with fistula formation to the trachea, esophagus, carotid artery or jugular vein. ${ }^{b}$ Other related deaths were caused by cachexia and sepsis. 'Severe adverse events observed in whole treatment course. P-value was estimated using Fisher's exact test and Kruskal-Wallis test appropriately. ${ }^{\mathrm{d}}$ Group of PFS > 10.5 months obtained statistically significant higher value or proportion compared to groups of Non-PD and PFS $\leq 10.5$ months. Abbreviations: IQR, interquartile range; PFS, progression free survival; OS, overall survival; $\mathrm{Cl}$, confidence interval; NA, not available or survival not reached yet.

$86.4 \%$ for sorafenib, and 5.9 months and $28.6 \%$ for Lenvatinib. $^{18}$ A study compared initial $24 \mathrm{mg} /$ day vs lower doses of 20, 14 and $10 \mathrm{mg} /$ day of lenvatinib for 30 Japanese DTC patients. The median PFS duration was 696 days (95\% CI: 318 not available) with response rate of $43 \%$ in the full-dose group, but the dose reduction was required in $93 \%$ of patients. The group with initial lower doses displayed a lower response rate (33\%) and dose reduction rate $67 \% .{ }^{19}$ Therefore, the proper dose of lenvatinib for RRDTC patients to achieve significant outcomes remains an important issue. Balancing the drug efficacy, AEs and quality of residual life (QoL) is always a great challenge for clinician. This real-world experience clearly demonstrated that low dose of lenvatinib treatment was well tolerated by our patients with less dose reduction, interruption, drug discontinuation and also achieved an acceptable outcome and QoL.
Generally, the recommended initial dose of lenvatinib is $24 \mathrm{mg}$ /day with a dose down-escalated to 20, 14 and finally $10 \mathrm{mg} /$ day gradually by AEs. However, the AEs rapidly resulted in dose discontinuation (82.4\%) and reduction (67\%) to initiate with $24 \mathrm{mg} / \mathrm{d}$ in SELECT trial. ${ }^{9,17}$ Based on a study evaluating tumor sizes treated with lenvatinib, drugtolerance-related treatment failure was highly concerned to initiate smaller doses of lenvatinib in an up-escalating titration schedule. $^{20}$ The impact of dose interruption was further analyzed in SELECT trial. All 261 patients experienced a dose interruption, but those with shorter dose interruptions (duration $<10 \%$ of total treatment course, median 19 days) had significantly longer PFS (not reached yet) versus 12.8 months (95\% CI: 9.3 16.5) in those with longer interruptions of lenvatinib (duration $\geq 10 \%$ of total treatment course, median 61 days). ${ }^{21}$ There was a distinct risk of accelerated tumor growth and sudden death following TKI drug withdrawal. ${ }^{18,}$ 


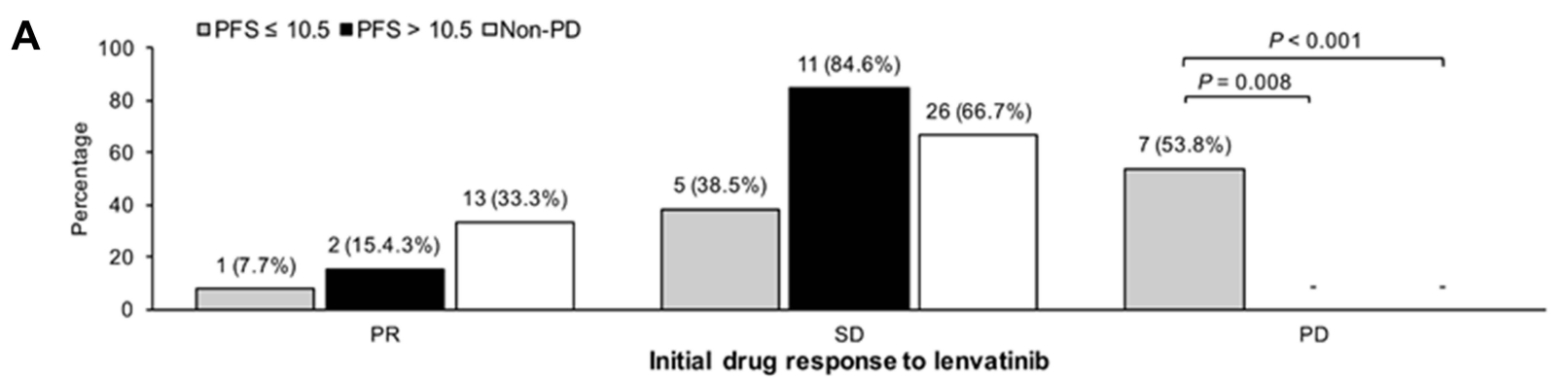

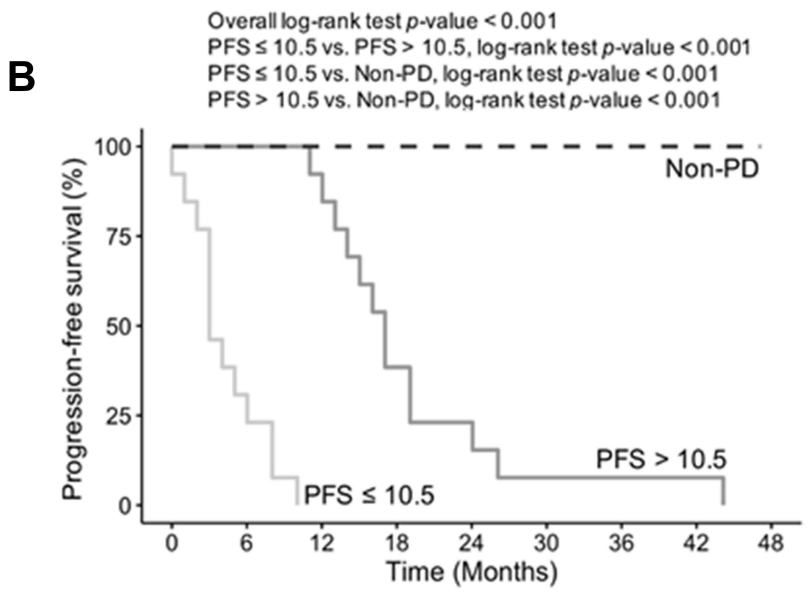

Number at risk

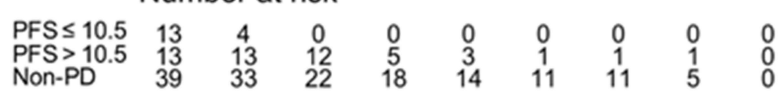

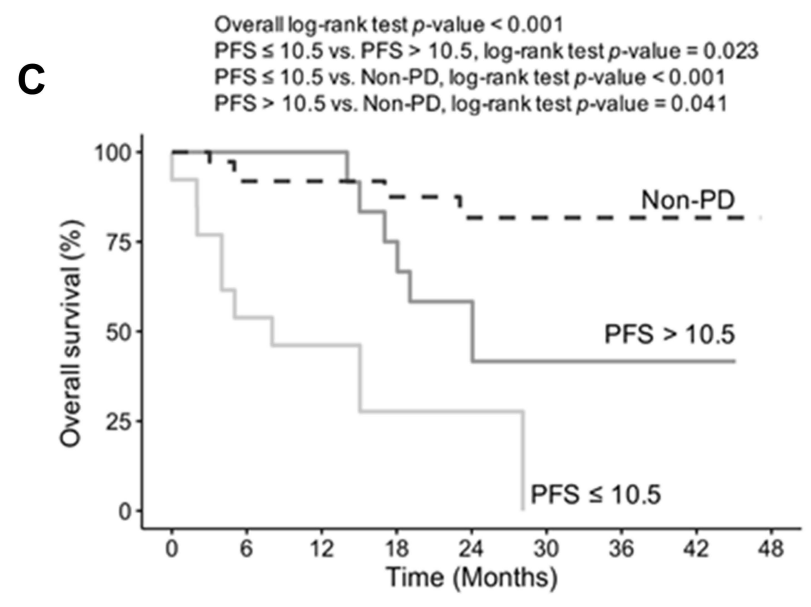

Number at risk

$\begin{array}{lccccccccc}\text { PFS }>10.5 & 13 & 4 & 0 & 0 & 0 & 0 & 0 & 0 & 0 \\ \text { Non-PD } & 39 & 33 & 12 & 5 & 3 & 1 & 1 & 1 & 0 \\ & 22 & 18 & 14 & 11 & 11 & 5 & 0\end{array}$

$\begin{array}{lccccccccc}\text { PFS } \leq 10.5 & 13 & 7 & 6 & 3 & 1 & 0 & 0 & 0 & 0 \\ \text { PFS }>10.5 & 13 & 13 & 12 & 9 & 7 & 5 & 5 & 4 & 0 \\ \text { Non-PD } & 39 & 33 & 22 & 18 & 14 & 11 & 11 & 5 & 0\end{array}$

Figure 2 Comparison of the initial drug response (A), median progression-free survival (PFS) (B), and overall survival (OS) (C) in PD patients with PFS $\leq 10.5$ months $(n=13)$, PFS $>10.5$ months $(n=13)$, and the patients with non-progressive disease (non-PD, $n=39)$. The $48-$ month PFS rate in the 3 groups were $0 \%, 0 \%$, and $100 \%$, respectively. The 48 -month OS rate in the 3 groups were $0 \%, 41.7 \%$ (95\% Cl: $21.3-81.4)$, and $81.7 \%$ (95\% Cl:67.5-98.9), respectively.

22-24 The flare-up phenomenon was rationally correlated to in vivo evidence from mice suggesting that tumor angiogenesis is halted by TKI therapy but rapid and full revascularization develops in 7 days from drug discontinuation. ${ }^{25,26}$ Thus, the option to continue TKI therapy as long as possible has been emphasized to avoid lethal tumor regrowth after drug discontinuation, especially in patients who progress slowly during treatment. ${ }^{18,26,27}$ Therefore, it is very important for timely management of $\mathrm{AE}$ to minimize the dose interruption and maximize the drug efficacy. Until now, no evidence documents the clinical efficacy of doses less than $10 \mathrm{mg} /$ day. Our study to initiate and maintain with low dose $(\sim 10 \mathrm{mg} /$ day $)$ of lenvatinib treatment displayed compatible drug efficacy with fewer high graded $\mathrm{AE}$ and less drug interruption.

So far, no biomarkers have been able to predict the response to lenvatinib even with many reports exploring potential predictive markers for drug response before treatment. $^{18}$ The lesion-based evaluation in a Korean study, with the mean dose of $16.0 \mathrm{mg} /$ day for 5 months, found that patients with rapid PD and shorter initial tumor doubling time could be predicted as better treatment responder but was still unable to obtain a longer PFS. Metastases to lung and brain occurred more frequently in lenvatinib treatment responders, but metastases to lymph nodes and bone were more prevalent in non-responders. ${ }^{28}$ Bone metastasis was regarded as a better independent negative prognostic factor for PFS than lung metastasis. $^{29}$ The SELECT trial found that lenvatinib responder could achieve a longer median PFS of 33.1 months than 7.9 months in non-responders. The median duration of response (DOR) for all lenvatinib responder was 30.0 months, but appeared shorter in patients with greater tumor burden, liver metastases or brain metastases. Previous study also suggests the initial response can sustain a prolonged response to treatment. ${ }^{30}$ Sub-analysis of the SELECT trial demonstrated tumor size response was also a predictive or prognostic factor. Tumor shrinkage by lenvatinib treatment was initially rapid $(24.7 \%$ within 2 months) and then gradually slowing down $(1.3 \%$ per month). The initial decrement of tumor size was 
a marginally significant positive predictor for PFS ( $\mathrm{P}=$ 0.06). ${ }^{20}$ The maximal benefit was also reported in patients with a better ECOG performance status. ${ }^{6}$ Our clinical experience obtained a similar finding. The initial drug response to lenvatinib at the first 3 months was highly correlated with the future prognosis well. A higher DCR achieved by initial lenvatinib treatment could significantly refer to a longer PFS and OS. In the PD groups, RRDTC patient with maximal dose exposure of RAI ( $\geq 600 \mathrm{mCi}$ ), or metastatic lesions to bone or other sites may be poor response indicators for low dose of lenvatinib treatment. Therefore, the drug response of the first 3 months could help physician to adjust the future treatment strategy.

In the SELECT trial, PD was reported in $6.9 \sim 8.4 \%$ of RRDTC patients even maintained with relative high dose of Lenvatinib. ${ }^{8,31}$ From this cohort, PD occurred in $10.8 \%$ of patients after initial treatment and then increased to $40.0 \%$ of patient at the end of follow-up. Also, PD occurred initially in $53.8 \%$ of group 1 patients, who had the shortest PFS and OS (3.0 months). The relative low-dose treatment may be a plausible factor in the rapid progression to death in our group 1 patients. However, intrinsic drug resistance to lenvatinib could also have had a critical role in the rapid PD, since most cancer patients may eventually develop an escape phenomenon or resistance to TKI therapy. ${ }^{18,26} \mathrm{Up}$ to date, there is still very little exploring the potential mechanisms of lenvatinib resistance. However, the pathways may involve activation of the cancer stem cells, which could become resistance to anti-angiogenic drugs through stimulation of alternative signaling pathways or upregulation of tumor cell receptors to promote tumor growth. To overcome or delay resistance to lenvatinib, combination with multiple drugs to achieve synergistic inhibition of different angiogenic or proliferative pathways may be a future strategy. ${ }^{18,32}$

Limitations of this study warrants mention, specifically the retrospective nature, conducted in a Taiwanese population only, without comparative dosage or a randomized design. Hence, our findings require validation by a prospective randomized trial to recruiting a larger and more general patient population.

In conclusion, low-dose lenvatinib treatment was well tolerated by RRDTC patients and displayed acceptable drug efficacy and outcomes with $89.2 \%$ DCR and a median PFS of 26.1 months. The relatively low dose of lenvatinib treatment may be more suitably applied in Asia.

\section{Abbreviations}

RRDTC, radioiodine-refractory differentiated thyroid carcinoma; PFS, progression-free survival; OS, overall survival; DCR, Disease control rate; PFS, Progressionfree survival; OS, Overall survival; DTC, differentiated thyroid carcinoma; RAI, radioiodine; TKI, multi-kinase inhibitor; AEs, adverse events; DCR, disease control rate; ECOG, Eastern Cooperative Oncology Group; RECIST, Response Evaluation Criteria in Solid Tumors; CTCAE, National Cancer Institute Common Terminology Criteria for Adverse Events; IOR, interquartile range; CIs, confidence intervals; PR, partial response; $\mathrm{SD}$, stable disease; $\mathrm{PD}$, progressive disease; NA, not available; QoL, quality of residual life; DOR, duration of response.

\section{Ethics Approval and Informed Consent}

This case series study was conducted and approved by the Institutional Review Boards of Medical centers of Kaohsiung Medical University (KMUHIRB-E (I)20190014) and Kaohsiung Chang-Gung Memorial Hospital (CGMHIRB No. 201801270B0).

\section{Acknowledgments}

The authors are grateful to Sin-Hua Moi, $\mathrm{PhD}$, Institute of Biotechnology and Chemical Engineering, I-Shou University, Kaohsiung, Taiwan, for her valuable assistance with statistical analysis.

\section{Author Contributions}

All authors made substantial contributions to conception and design, acquisition of data, or analysis and interpretation of data; took part in drafting the article or revising it critically for important intellectual content; agreed to submit to the current journal; gave final approval of the version to be published; and agree to be accountable for all aspects of the work.

\section{Funding}

This research did not receive any specific grant from funding agencies in the public, commercial, or not-forprofit sectors. 


\section{Disclosure}

All authors declared that there is no conflict of interest that could be perceived as prejudicing the impartiality of the research reported.

\section{References}

1. Cabanillas ME, McFadden DG, Durante C. Thyroid cancer. Lancet. 2016;388(10061):2783-2795. doi:10.1016/S0140-6736(16)30172-6

2. Tamai T, Hayato $S$, Hojo $S$, et al. Dose finding of lenvatinib in subjects with advanced hepatocellular carcinoma based on population pharmacokinetic and exposure-response analyses. J Clin Pharmacol. 2017;57(9):1138-1147. doi:10.1002/jcph.917

3. Haugen BR, Sherman SI. Evolving approaches to patients with advanced differentiated thyroid cancer. Endocr Rev. 2013;34 (3):439-455. doi:10.1210/er.2012-1038

4. Schmidbauer B, Menhart K, Hellwig D, Grosse J. Differentiated thyroid cancer - treatment: state of the art. Int J Mol Sci. 2017;18 (6): 1292 . doi: $10.3390 / \mathrm{ijms} 18061292$

5. Pacini F. Which patient with thyroid cancer deserves systemic therapy and when? Best Pract Res Clin Endocrinol Metab. 2017;31 (3):291-294. doi:10.1016/j.beem.2017.08.001

6. Berdelou A, Lamartina L, Klain M, Leboulleux S, Schlumberger M. Treatment of refractory thyroid cancer. Endocr Relat Cancer. 2018;25(4):R209-R223. doi:10.1530/ERC-17-0542

7. Cabanillas ME, Habra MA. Lenvatinib: role in thyroid cancer and other solid tumors. Cancer Treat Rev. 2016;42:47-55. doi:10.1016/j. ctrv.2015.11.003

8. Schlumberger M, Tahara M, Wirth LJ, et al. Lenvatinib versus placebo in radioiodine-refractory thyroid cancer. $N$ Engl $J$ Med. 2015;372(7):621-630. doi:10.1056/NEJMoa1406470

9. Costa R, Carneiro BA, Chandra S, et al. Spotlight on lenvatinib in the treatment of thyroid cancer: patient selection and perspectives. Drug Des Devel Ther. 2016;10:873-884. doi:10.2147/DDDT.S93459

10. Nair A, Lemery SJ, Yang J, et al. FDA approval summary: lenvatinib for progressive, radio-iodine-refractory differentiated thyroid cancer. Clin Cancer Res. 2015;21(23):5205-5208. doi:10.1158/1078-0432. CCR-15-1377

11. Ferrari SM, Ruffilli I, Centanni M, et al. Lenvatinib in the therapy of aggressive thyroid cancer: state of the art and new perspectives with patents recently applied. Recent Pat Anticancer Drug Discov. 2018;13(2):201-208. doi:10.2174/1574892813666180220110729

12. Berdelou A, Borget I, Godbert Y, et al. Lenvatinib for the treatment of radioiodine-refractory thyroid cancer in real-life practice. Thyroid. 2018;28(1):72-78. doi:10.1089/thy.2017.0205

13. Kiyota N, Schlumberger M, Muro K, et al. Subgroup analysis of Japanese patients in a Phase 3 study of lenvatinib in radioiodine-refractory differentiated thyroid cancer. Cancer Sci. 2015;106(12):1714-1721. doi:10.1111/cas.12826

14. Nagahama M, Ozeki T, Suzuki A, et al. Association of lenvatinib trough plasma concentrations with lenvatinib-induced toxicities in Japanese patients with thyroid cancer. Med Oncol. 2019;36(5):39. doi:10.1007/s12032-019-1263-3

15. Brose MS, Nutting CM, Jarzab B, et al. Sorafenib in radioactive iodine-refractory, locally advanced or metastatic differentiated thyroid cancer: a randomised, double-blind, phase 3 trial. Lancet. 2014;384(9940):319-328. doi:10.1016/S0140-6736(14)60421-9

16. Yu ST, Ge JN, Luo JY, Wei ZG, Sun BH, Lei ST. Treatment-related adverse effects with TKIs in patients with advanced or radioiodine refractory differentiated thyroid carcinoma: a systematic review and meta-analysis. Cancer Manag Res. 2019;11:1525-1532. doi:10.2147/ cmar.s191499
17. Capdevila J, Newbold K, Licitra L, et al. Optimisation of treatment with lenvatinib in radioactive iodine-refractory differentiated thyroid cancer. Cancer Treat Rev. 2018;69:164-176. doi:10.1016/j. ctrv.2018.06.019

18. Iwasaki H, Yamazaki H, Takasaki H, et al. Treatment outcomes of differentiated thyroid cancer with distant metastasis improve by tyrosine kinase inhibitors. Oncol Lett. 2019;17(6):5292-5300. doi:10.3892/ol.2019.10180

19. Yamazaki H, Iwasaki H, Takasaki H, et al. Efficacy and tolerability of initial low-dose lenvatinib to treat differentiated thyroid cancer. Medicine. 2019;98(10):e14774. doi:10.1097/md.0000000000014774

20. Robinson B, Schlumberger M, Wirth LJ, et al. Characterization of tumor size changes over time from the phase 3 study of lenvatinib in thyroid cancer. J Clin Endocrinol Metab. 2016;101(11):4103-4109. doi:10.1210/jc.2015-3989

21. Tahara M, Brose MS, Wirth LJ, et al. Impact of dose interruption on the efficacy of lenvatinib in a phase 3 study in patients with radioiodine-refractory differentiated thyroid cancer. Eur $J$ Cancer. 2019;106:61-68. doi:10.1016/j.ejca.2018.10.002

22. Yun KJ, Kim W, Kim EH, et al. Accelerated disease progression after discontinuation of sorafenib in a patient with metastatic papillary thyroid cancer. Endocrinol Metab. 2014;29(3):388-393. doi:10.3803/EnM.2014.29.3.388

23. Uchida T, Yamaguchi H, Nagamine K, et al. Rapid pleural effusion after discontinuation of lenvatinib in a patient with pleural metastasis from thyroid cancer. Endocrinol Diabetes Metab Case Rep. Epub 2019 Mar 18. doi:10.1530/EDM-18-0158

24. Markman M. Lenvatinib real-life experience. Oncology. 2019;97 (4):189-191. doi:10.1159/000501641

25. Mancuso MR, Davis R, Norberg SM, et al. Rapid vascular regrowth in tumors after reversal of VEGF inhibition. J Clin Invest. 2006;116 (10):2610-2621. doi:10.1172/JCI24612

26. Matrone A, Valerio L, Pieruzzi L, et al. Protein kinase inhibitors for the treatment of advanced and progressive radiorefractory thyroid tumors: from the clinical trials to the real life. Best Pract Res Clin Endocrinol Metab. 2017;31(3):319-334. doi:10.1016/j. beem.2017.06.001

27. Viola D, Valerio L, Molinaro E, et al. Treatment of advanced thyroid cancer with targeted therapies: ten years of experience. Endocr Relat Cancer. 2016;23(4):R185-R205. doi:10.1530/ERC-15-0555

28. Lee EK, Kim S-M, Kim BH, et al. Lesion-based evaluation predicts treatment response to lenvatinib for radioactive iodine-refractory differentiated thyroid cancer: a Korean multicenter retrospective study. Thyroid. 2019;29(12):1811-1819. doi:10.1089/thy.2019.0022

29. Suzuki C, Kiyota N, Imamura Y, et al. Exploratory analysis of prognostic factors for lenvatinib in radioiodine-refractory differentiated thyroid cancer. Head Neck. 2019;41(9):3023-3032. doi:10.1002/hed.25784

30. Gianoukakis AG, Dutcus CE, Batty N, Guo M, Baig M. Prolonged duration of response in lenvatinib responders with thyroid cancer. Endocr Relat Cancer. 2018;25(6):699-704. doi:10.1530/erc-18-0049

31. Kiyota N, Robinson B, Shah M, et al. Defining radioiodine-refractory differentiated thyroid cancer: efficacy and safety of lenvatinib by radioiodine-refractory criteria in the SELECT TRIAL. Thyroid. 2017;27(9):1135-1141. doi:10.1089/thy.2016.0549

32. Tuttle RM, Brose MS, Grande E, Kim SW, Tahara M, Sabra MM. Novel concepts for initiating multitargeted kinase inhibitors in radioactive iodine refractory differentiated thyroid cancer. Best Pract Res Clin Endocrinol Metab. 2017;31(3):295-305. doi:10.1016/j. beem.2017.04.014 


\section{Publish your work in this journal}

Cancer Management and Research is an international, peer-reviewed open access journal focusing on cancer research and the optimal use of preventative and integrated treatment interventions to achieve improved outcomes, enhanced survival and quality of life for the cancer patient.

The manuscript management system is completely online and includes a very quick and fair peer-review system, which is all easy to use. Visit http://www.dovepress.com/testimonials.php to read real quotes from published authors.

Submit your manuscript here: https://www.dovepress.com/cancer-management-and-research-journa 